\title{
Uma visão cinesiológico-funcional sobre a dor sexual no puerpério: revisão
}

\author{
Sexual pain after childbirth: review
}

Ariana F. dos S. Bartolomey Mendes

Paula Ferrarezi Brunazzo Fernanda Morais Silva

Brenda Beatriz Silva Monteiro ${ }^{I}$ Erica Feio Carneiro Nunes ${ }^{I}$ Gustavo Fernando Sutter LATORRE ${ }^{\text {II }}$

${ }^{\mathrm{I}}$ Universidade do Estado do Pará, Belém/PA - Brasil

"I Gustavo Latorre Cursos
RESUMo - Introdução: Muitas mulheres acreditam ser comum apresentar dor durante relação sexual, principalmente no puerpério, não relatando isso ao ginecologista ou a um profissional da saúde, favorecendo a instalação de dor crônica, muito embora já exista tratamento e prevenção para a dor sexual feminina com fisioterapia pélvica. Objetivo: discutir a incidência e fatores de risco associados à dor sexual em puérperas, de um ponto de vista cinesiológico-funcional. Método: revisão eletrônica das bases de dados LILACS, PubMed, PEDro e SciELO utilizando os descritores "sexual pain" e "puerperal". Resultados: Foram encontrados 66 artigos, destes 13 artigos preencheram os critérios de inclusão e exclusão. A prevalência de dor sexual foi de 30$50 \%$ em puérperas, está presente entre 6 e 12 meses pós-parto, é causada por consequências do puerpério e gera grande impacto na vida destas mulheres. Fatores de risco para a dor sexual são a via de parto, amamentação e sequelas mecânicas especialmente do parto vaginal, instrumentalizado ou com laceração. Conclusão: A dor sexual pode estar presente desde a gestação e se tornar crônica no puerpério. Sequelas funcionais são verificadas nestas mulheres, e podem ser tratadas e prevenidas por fisioterapia pélvica.

Palavras-chave: Puerpério; Saúde Sexual; Fisioterapia.

AbstraCt - Introduction: Many women consider pain during sexual intercourse an usual thing, especially in the postpartum period. They do not report this condition to the health care professional, favoring the onset of chronic pain, although there is already treatment and prevention for female sexual pain with pelvic physiotherapy. Aims: Discuss the incidence and risk factors associated with sexual pain in puerperal women, from a kinesiological-functional point of view. Method: electronic review of LILACS, PubMed, PEDro and SciELO databases using the descriptors "sexual pain" and "puerperal". Results: found 66 articles, 13 surviving the inclusion and exclusion criteria. The prevalence of sexual pain was $30-50 \%$ in postpartum women. It is present between 6 and 12 months postpartum, and caused by consequences of the puerperium, having great impact on quality of life. Risk factors are the way of delivery, breastfeeding and mechanical sequelae, especially through vaginal delivery, instrumented delivery or laceration. Conclusion: Sexual pain may be present since pregnancy and may be chronic in the postpartum period. Functional sequelae are found in these women, and can be treated and prevented by pelvic physiotherapy.

Keywords: Puerperium; Sexual Health; Physiotherapy. 


\section{INTRODUÇÃO}

O puerpério é responsável por importantes modificações na vida da mulher, da família e do casal, incluindo alterações hormonais, anatômicas, psicológicas e sociais. ${ }^{1}$ Durante esse período é comum que as puérperas relatem algum tipo de dor e/ou desconforto durante a relação sexual, situação que pode ocorrer desde a primeira relação sexual e persistir em até um ano ou mais, caracterizando disfunção sexual crônica. ${ }^{2}$

A disfunção sexual é oriunda da desordem de uma ou mais das fases do ciclo da resposta sexual, sendo desejo, excitação, lubrificação, orgasmo, satisfação ou dor/ desconforto associado ao ato sexual. ${ }^{3} \mathrm{~A}$ disfunção do assoalho pélvico está relacionada às disfunções sexuais femininas, sendo especialmente o parto vaginal responsável por impactos importantes sobre a função do assoalho pélvico. ${ }^{4}$

A falta de informação oferecida para a gestante, o desejo da mãe de evitar dor e sofrimento, a crença de um processo mais fácil com menores riscos, a possibilidade de marcar uma data ou realizar laqueadura, o controle maior sobre o nascimento e também pelo medo relacionado ao parto e suas possíveis complicações são as razões pelas quais algumas gestantes geralmente optam pelo parto cesáreo. ${ }^{4}$ Já as opções pelo parto vaginal ocorrem por causa do menor tempo de recuperação de suas atividades de vida diária, menor dor no pós-parto e do protagonismo vivenciado pela mulher. ${ }^{5}$ Todavia, nesta discussão, é importante ressaltar que o parto cesáreo não previne os problemas comuns do par- to vaginal, como minimização de episiotomias ou risco de laceração, uma vez que grande parte dos partos vaginais acontece sem estas sequelas. ${ }^{6}$

A dispareunia, conhecida como coito doloroso, é uma complicação comum no puerpério imediato, mas pode persistir por mais de um ano após o parto. ${ }^{7}$ Geralmente, a dispareunia está relacionada às condições multifatoriais como fatores físicos, comportamentais ou psicológicos, os quais vão dificultar o diagnóstico e tratamento precoce. ${ }^{8}$

A dispareunia no puerpério tem causas principalmente obstétricas e ginecológicas, as quais impactam negativamente sobre a qualidade de vida dessas mulheres. A laceração e o parto instrumentalizado são fatores para a ocorrência de dor genital, impactando negativamente sobre os vários domínios da vida sexual da mulher. ${ }^{9}$

Todavia, segue na literatura, ainda hoje, controvérsia a respeito da correlação da via de parto com a presença de dispareunia e do parto cirúrgico como, por exemplo, auxiliado por episiotomia, como forma protetora ou não de disfunção do assoalho pélvico. Ao considerarmos ainda que hoje a fisioterapia pélvica emerge fortemente no tratamento da dor sexual feminina ${ }^{10,11}$, parece fundamental que as possíveis sequelas da gestação e parto sobre a função sexual feminina, particularmente quanto à presença de dor sexual, seja entendida e discutida sob o prisma cinesiológico-funcional da fisioterapia pélvica. É esta discussão, por fim, o objetivo do estudo: discutir a incidência e fatores de risco associados à dor sexual em puérperas, de um ponto de vista cinesiológico-funcional. 


\section{Metodologia}

Trata-se de uma revisão da literatura a partir de buscas nas bases de dados eletrônicas LILACS, PubMed, PEDro e SciELO.

$O$ desenvolvimento desta pesquisa ocorreu em duas etapas. $1^{\text {a }}$ etapa - Embasamento Teórico: $\mathrm{O}$ fundamento teórico do estudo foi pesquisado em banco de dados como Scientific Electronic Library Online (SciELO), Literatura Latino Americana e do Caribe em Ciências da Saúde (LILACS) e National Library of Medicine (PUBMED). Esse levantamento bibliográfico ocorreu durante os meses de agosto a novembro de 2020. Foram utilizados os descritores: "Puerpério" or "Pós-parto" and "Saúde Sexual" and "Fisioterapia". Na $2^{\text {a }}$ etapa, foram incluídos estudos transversais que associassem a dor sexual ao puerpério publicados entre os anos de 2013 e 2019, na língua portuguesa e inglesa. Foram excluídos artigos de revisão, estudos realizados em animais e artigos duplicados nas bases de dados.

O processo de seleção do estudo foi realizado por dois revisores, e envolveu a triagem dos títulos e leitura dos resumos, após o qual os artigos potencialmente relevantes foram obtidos no texto completo para uma análise mais aprofundada dos critérios de elegibilidade. Um terceiro revisor foi consultado em casos de desacordo, e uma decisão foi tomada por consenso.
Os dados coletados foram: autores, descrição da amostra (idade, tipo de parto) instrumento de avaliação, resultados e as conclusões do estudo.

\section{Resultados}

A partir da estratégia de busca, 66 artigos foram encontrados, contudo, após a aplicação dos critérios de elegibilidade, 13 foram considerados elegíveis para análise. O fluxo de estudos por meio do processo de seleção é apresentado na Figura 1.

A base PubMed retornou um total de 62 artigos, sendo incluídos 13 artigos. Dos artigos não incluídos, 17 artigos eram de revisão de literatura, 18 artigos não abordavam ou não avaliavam dispareunia, 14 artigos não abordavam sobre puerpério e não abordavam ou avaliavam dispareunia. Quanto às exclusões, uma estava duplicada em outras bases de dados. A base de dados PEDro não contabilizou nenhum artigo com as palavras-chave descritas. A base SciELO retornou apenas um artigo que não foi incluído por não analisar a dor na relação sexual. A base LILACS retornou 3 artigos, nenhum foi incluído pois dois eram de língua espanhola e também não correlacionavam a dispareunia com o puerpério e um artigo era de revisão sistemática.

O tamanho das amostras variou de 451 no estudo realizado por Persico et al. ${ }^{29}$, a 70 por Demaria et al. ${ }^{13}$. A descrição completa dos estudos é apresentada no Quadro 1. 


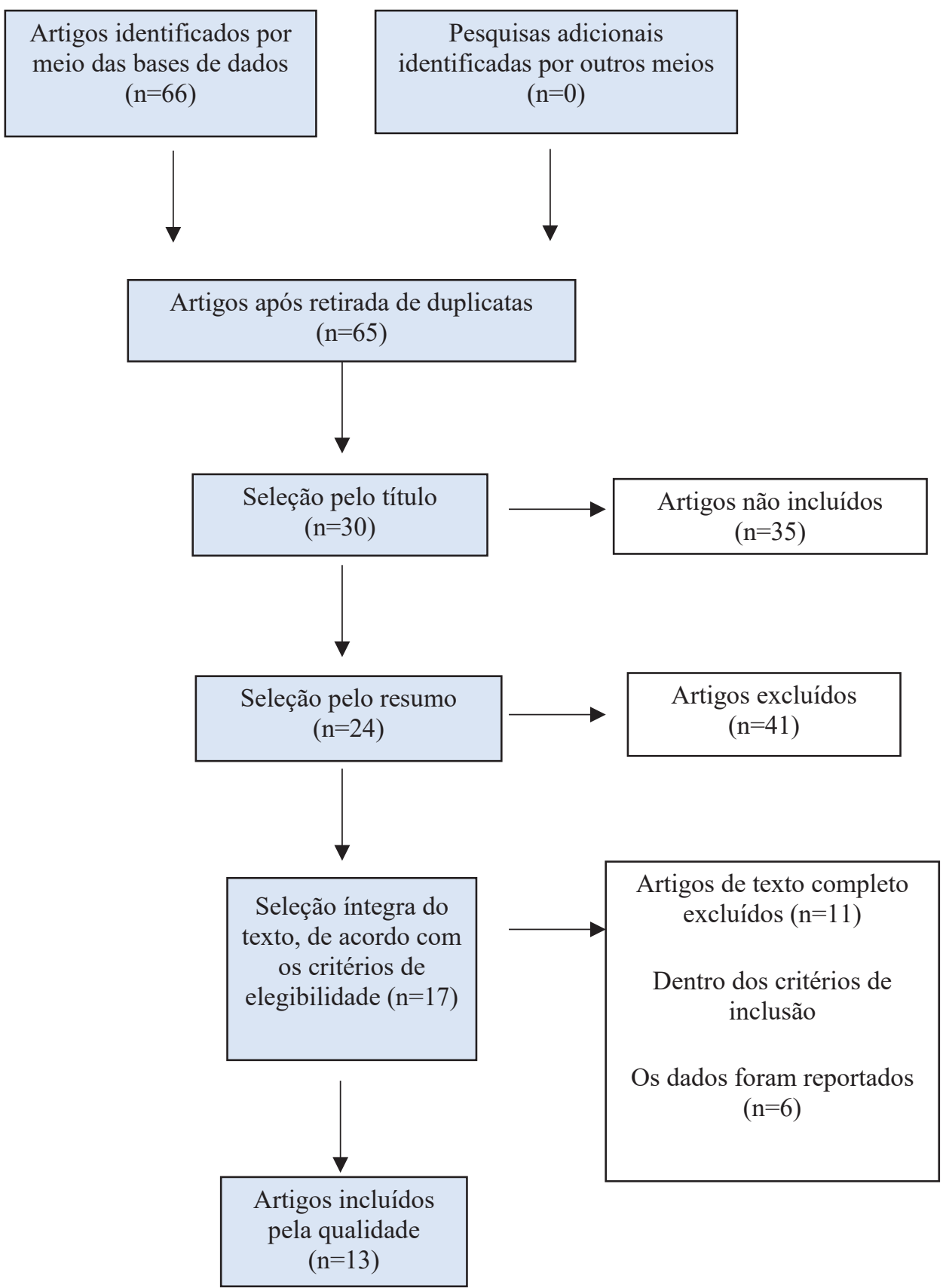

Figura 1: Fluxograma com o processo de seleção, inclusão e exclusão dos artigos primários. 
Os achados dos artigos falam sobre a incidência e fatores de risco associados à dor em puérperas, relacionando também o parto vaginal e parto cesárea com a função sexual. Além disso, é oportuno frisar que os partos vaginais estiveram mais relacionados à dor sexual, sobretudo quando relacionado à utilização de instrumentos como fórceps.

Quadro 1. Resumo dos estudos incluídos.

\begin{tabular}{|c|c|c|c|c|}
\hline Autor/ano & $\begin{array}{l}\text { Amostra (características da } \\
\text { amostra) }\end{array}$ & $\begin{array}{l}\text { Instrumento } \\
\text { de avaliação }\end{array}$ & Resultados & Conclusão \\
\hline Persico et al. ${ }^{30}$ & $\begin{array}{l}\text { n: } 451 \text { mulheres; } \\
\text { Primíparas: } 239 \text {; } \\
\text { Multíparas: } 212 .\end{array}$ & ENV e ECV & $\begin{array}{l}\text { - A prevalência de dor foi encontrada em } 88,2 \% \\
\text { no 1a dia PP } \\
\text { - } 62.3 \% 1 \text { semana PP; } 8 \% \text { em } 7 \text { semanas; e 0.7\% } \\
\text { em } 6 \text { meses. } \\
\text { - Dispareunia em primíparas: } 35,6 \% \text { em } 7 \\
\text { semanas PP. } 23,3 \% \text { em } 6 \text { meses. } \\
\text { - Dispareunia em multíparas: } 29,1 \% \text { em } 7 \\
\text { semanas. 10,1\% em } 6 \text { meses. } \\
\text { - Dispareunia em episiotomia: } 40.4 \% \text { em } 7 \\
\text { semanas PP. } 27 \% \text { em } 6 \text { meses PP. } \\
\text { - Dispareunia na laceração: } 32.9 \% \text { em } 7 \\
\text { semanas. 17.8\% em } 6 \text { meses PP. } \\
\text { - Dispareunia em períneo íntegro: } 25.6 \% \text { em } 7 \\
\text { semanas PP. 4,9\% em } 6 \text { meses PP. }\end{array}$ & $\begin{array}{l}\text { A dor perineal está } \\
\text { presente em } 95 \% \text { das } \\
\text { mulheres que sofreram } \\
\text { trauma perineal. Foi } \\
\text { demonstrado que } \\
\text { mulheres com PVE } \\
\text { e trauma perineal } \\
\text { mínimo ou ausente } \\
\text { terão melhores } \\
\text { recuperações: menos } \\
\text { dor, mais músculos } \\
\text { perineais tônicos, FS } \\
\text { melhorada e menores } \\
\text { taxas de DPP. }\end{array}$ \\
\hline Dabiri et al. ${ }^{14}$ & $\begin{array}{l}\text { n: } 248 \text { mulheres; } \\
\text { Idade: } 18 \text { a } 35 \text { anos; } \\
\text { PV: } 156 \text {; } \\
\text { Episiotomia: } 156 ; \\
\text { PC: } 96 .\end{array}$ & FSFI & $\begin{array}{l}\text { - Dor, excitação, lubrificação, orgasmo e } \\
\text { satisfação são afetados em mulheres que fizeram } \\
\text { episiotomia (156). } \\
\text { - Mulheres que tiveram episiotomia na região } \\
\text { mediolateral, tiveram diminuição do desejo } 6 \\
\text { meses PP quando comparado a pré-gravidez. }\end{array}$ & $\begin{array}{l}\text { O MP parece ser } \\
\text { uma questão crucial } \\
\text { para a FS no PP, } \\
\text { principalmente em } \\
\text { mulheres que tiveram } \\
\text { episiotomia. }\end{array}$ \\
\hline $\begin{array}{l}\text { Chayachinda } \\
\text { et al. }{ }^{25}\end{array}$ & $\begin{array}{l}\text { n: } 93 \text { mulheres sexualmente } \\
\text { ativas; } \\
\text { Idade: } 18 \text { a } 37 \text { anos. }\end{array}$ & EVA e FSFI & $\begin{array}{l}\text { - Após } 3 \text { meses, } 30.1 \% \text { das participantes } \\
\text { relataram dispareunia. } \\
\text { - Não houve associação entre dispareunia e } \\
\text { dispareunia pré-gestacional, circunferência } \\
\text { da cabeça do recém-nascido, peso ao nascer e } \\
\text { aleitamento materno. } \\
\text { - Em } 3,6 \text { e } 12 \text { meses, a dispareunia foi de } 66.7 \% \text {, } \\
31 \%, 14.9 \% \text {, respectivamente. } \\
\text { - Não houve diferença nas pontuações em todos } \\
\text { os domínios aos } 3 \text { e } 12 \text { meses entre as mulheres } \\
\text { com e sem dispareunia. } \\
\text { - Aos } 6 \text { meses, àquelas sem dispareunia } \\
\text { tiveram melhores pontuações em dor, orgasmo, } \\
\text { satisfação e escore total. }\end{array}$ & $\begin{array}{l}\text { A prevalência de } \\
\text { dispareunia em } 3 \\
\text { meses e as mudanças } \\
\text { nos escores de FSFI } \\
\text { durante o primeiro } \\
\text { ano PP foram os } \\
\text { principais desfechos. A } \\
\text { dispareunia aos } 3 \text { meses } \\
\text { é comum em mulheres } \\
\text { primíparas com } \\
\text { episiotomia. Àquelas } \\
\text { com dispareunia têm } \\
\text { uma RRS lenta da FS } \\
\text { normal. }\end{array}$ \\
\hline $\begin{array}{l}\text { Kabakian- } \\
\text { Khasholian } \\
\text { et al. }{ }^{19}\end{array}$ & $\begin{array}{l}\text { n: } 238 \text { mulheres; } \\
\text { Idade: > } 18 \text { anos; } \\
\text { PV: } 134 ; \\
\text { PC: } 104\end{array}$ & Questionário & $\begin{array}{l}\text { - } 67 \% \text { relataram sentir dor durante a relação } \\
\text { sexual PP e } 72,3 \% \text { não procuraram atendimento. } \\
\text { - PC aumenta as chances de sentir dor durante a } \\
\text { relação sexual no período PP. }\end{array}$ & $\begin{array}{l}\text { PC pode aumentar } \\
\text { as chances de relatar } \\
\text { dor durante a relação } \\
\text { sexual PP entre } \\
\text { mulheres primíparas. }\end{array}$ \\
\hline
\end{tabular}




\begin{tabular}{|c|c|c|c|c|}
\hline $\begin{array}{l}\text { McDonald et } \\
\text { al. } .^{15}\end{array}$ & $\begin{array}{l}\text { n: } 1507 \text { mulheres; } \\
\text { Idade: } 18 \text { a } 35 \text { anos; } \\
\text { PVE: } 109 ; \\
\text { Sutura/episiotomia: } 256 \text {; } \\
\text { PI: } 152 ; \\
\text { PC: } 990 \text {. }\end{array}$ & Questionário & $\begin{array}{l}\text { - } 89 \% \text { das mulheres relataram problemas de } \\
\text { saúde sexual nos primeiros } 3 \text { meses PP. } \\
\text { - } 51 \% \text { continuaram relatando perda de interesse } \\
\text { em sexo aos } 12 \text { meses PP e cerca de } 30 \% \\
\text { relataram dor persistente. } \\
\text { - PC: mais problemas sexuais. } \\
\text { - } 44,7 \% \text { dispareunia em } 3 \text { meses PP; } 43,4 \% \text {; em } \\
6 \text { meses PP, } 28,1 \% \text { em } 12 \text { meses PP e } 23,4 \% \text { em } \\
18 \text { meses. }\end{array}$ & $\begin{array}{l}\text { Questões de saúde } \\
\text { sexual são comuns } \\
\text { PP. Apesar do contato } \\
\text { frequente com os } \\
\text { profissionais, as } \\
\text { mulheres raramente } \\
\text { discutiam sobre a FS. }\end{array}$ \\
\hline 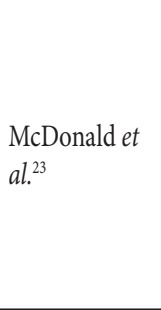 & $\begin{array}{l}\text { n: } 1507 \text { nulíparas; } \\
\text { Idade: } 18 \text { a } 40 \text { anos; } \\
\text { PV: } 1075 ; \\
\text { Episiotomia: } 228 ; \\
\text { Extração à vácuo: } 149 ; \\
\text { Fórceps: } 150 ; \\
\text { PVE: } 5 \text {; } \\
\text { PC: } 432 \text {. }\end{array}$ & Questionário & $\begin{array}{l}\text { - } 1211 \text { RRS aos } 18 \text { meses PP; dessas, } 289 \\
\text { relataram dispareunia. } \\
\text { - Aos } 3 \text { meses pós parto, } 970 \text { haviam RRS, } 1165 \\
\text { aos } 6 \text { meses pós parto. } \\
\text { - A maioria das mulheres (961) que RRS } 12 \\
\text { meses PP, experimentou dor durante o primeiro } \\
\text { sexo vaginal. } \\
\text { - A dispareunia foi relatada por } 431 \text { mulheres. }\end{array}$ & $\begin{array}{l}\text { A intervenção } \\
\text { obstétrica } \\
\text { (especificamente } \\
\text { extração à vácuo e } \\
\text { cesariana) contribui } \\
\text { para dispareunia } \\
\text { persistente após } 18 \\
\text { meses PP. }\end{array}$ \\
\hline Ahmed et al. ${ }^{20}$ & $\begin{array}{l}\text { n: } 156 \text { mulheres; } \\
\text { Grupo Controle (episiotomia } \\
\text { ou pequenas lacerações): } 100 ; \\
\text { Grupo Estudado (lacerações } \\
\text { perineais de } 3^{\circ} \text { e } 4^{\circ} \text { grau após } \\
\text { parto vaginal): } 56 \text {; } \\
\text { Idade Grupo Controle: } 31 \text { a } \\
37 \text { anos; } \\
\text { Idade Grupo Estudado: } 29 \text { a } \\
37 \text { anos. }\end{array}$ & FSFI & $\begin{array}{l}\text { - Em ambos os grupos (controle e estudado) } \\
\text { os escores médios totais do FSFI foram } \\
\text { significativamente diferentes aos } 6 \text { meses PP. } \\
\text { - Após } 12 \text { meses, apesar da ligeira melhora, a FS } \\
\text { foi menor no grupo estudado em comparação } \\
\text { com o grupo controle. } \\
\text { - As mulheres do grupo estudado mostraram } \\
\text { diminuições significativas nas pontuações } \\
\text { de desejo, excitação, lubrificação, satisfação, } \\
\text { orgasmo e dor aos } 12 \text { meses PP. }\end{array}$ & $\begin{array}{l}\text { Lacerações perineais } \\
\text { de alto grau afetam } \\
\text { negativamente a } \\
\text { FS até } 1 \text { ano PP. A } \\
\text { avaliação da FS e } \\
\text { o aconselhamento } \\
\text { adequado são } \\
\text { necessários para } \\
\text { mulheres com lacerais } \\
\text { perineais. }\end{array}$ \\
\hline $\begin{array}{l}\text { McDonald } \\
\text { et al. }\end{array}$ & $\begin{array}{l}\text { n: } 1507 \text { nulíparas; } \\
\text { Idade: > } 18 \text { anos; } \\
\text { PV: } 1139 ; \\
\text { PC: } 368 ; \\
\text { Fórceps ou extração à Vácuo: } \\
\text { 267; }\end{array}$ & $\begin{array}{l}\text { Escala de dor } \\
\text { de McGill }\end{array}$ & $\begin{array}{l}\text { - Dor no primeiro sexo vaginal PP nos } \\
\text { primeiros } 12 \text { meses: } 1122 \text {. } \\
\text { - A dispareunia reduziu ao longo do tempo, } \\
\text { de } 44.7 \% \text { em três meses PP para } 22.6 \% \text { aos } 18 \\
\text { meses PP. } \\
\text { - PC: maior probabilidade de relatar dispareunia } \\
\text { mais intensa há } 6 \text { meses PP. } \\
\text { - RRS após } 3 \text { meses: } 970 \text {. Após } 6 \text { meses: } 1165 ; 12 \\
\text { meses PP: } 1202 \text {. }\end{array}$ & $\begin{array}{l}\text { A dispareunia PP } \\
\text { diminui com o tempo, } \\
\text { mas persiste além de } \\
12 \text { meses em um em } \\
\text { cada cinco mulheres. A } \\
\text { cesariana parece estar } \\
\text { associada à dispareunia } \\
\text { mais intensa. Cerca } \\
\text { de } 10 \% \text { das mulheres } \\
\text { relataram dispareunia } \\
\text { como angustiante e } \\
\text { horrível. }\end{array}$ \\
\hline $\begin{array}{l}\text { Necesalova } \\
\text { et al. }{ }^{33}\end{array}$ & $\begin{array}{l}\text { n: } 648 \text { primíparas; } \\
\text { Episiotomia médio-lateral: } \\
306 ; \\
\text { Episiotomia lateral: } 342 .\end{array}$ & FSFI & $\begin{array}{l}\text { - Em } 3 \text { meses PP, } 71.3 \% \text { do grupo com } \\
\text { episiotomia médio-lateral relatou dispareunia, } \\
\text { contra } 70.4 \% \text { do grupo episiotomia lateral. } \\
\text { - Em } 6 \text { meses PP, } 50.7 \% \text { do grupo episiotomia } \\
\text { médio-lateral relatou dispareunia, contra } 55.4 \% \\
\text { do grupo episiotomia lateral. } \\
\text { - Não houve diferença significativa entre os } \\
\text { grupos quanto à satisfação sexual. }\end{array}$ & $\begin{array}{l}\text { A qualidade de vida } \\
\text { sexual e percepção } \\
\text { da dor perineal após } \\
\text { a episiotomia médio- } \\
\text { lateral é equivalente a } \\
\text { episiotomia lateral. }\end{array}$ \\
\hline
\end{tabular}




\begin{tabular}{|c|c|c|c|c|}
\hline Lagaert et al. ${ }^{12}$ & $\begin{array}{l}\text { n: } 78 \text { mulheres; } \\
\text { Idade: } 21 \text { a } 40 \text { anos; } \\
\text { PV: } 63 \\
\text { Espontâneo: } 55 \text {; } \\
\text { Fórceps/auxílio de Vácuo: } 8 \text {; } \\
\text { Laceração: } 21 \text {; } \\
\text { Episiotomia: } 34 ; \\
\text { PC: } 15 \text {. }\end{array}$ & FSFI & $\begin{array}{l}\text { - Dispareunia: mais de } 50 \% \text { tiveram em seis } \\
\text { semanas PP, diminuindo para } 40,7 \% \text { seis meses } \\
\text { PP. } \\
\text { - A dor foi associada ao aleitamento materno e à } \\
\text { primiparidade. } \\
\text { - As primíparas apresentaram mais dor do que } \\
\text { as multíparas. }\end{array}$ & $\begin{array}{l}\text { A dispareunia é } \\
\text { uma doença muito } \\
\text { comum PP. O PV } \\
\text { está mais associado } \\
\text { à dispareunia, sendo } \\
\text { mais frequente em } \\
\text { primíparas. }\end{array}$ \\
\hline Nolens et al..$^{32}$ & $\begin{array}{l}\text { n: } 646 \text { puérperas; } \\
\text { Partos com extração á vácuo: } \\
289 ; \\
\text { PC: } 357 \text {; } \\
\text { Episiotomia: } 94 \text {. }\end{array}$ & $\begin{array}{l}\text { Questionário } \\
\text { SF-12 }\end{array}$ & $\begin{array}{l}\text { - Aos } 6 \text { meses, quase todas haviam RRS em } \\
\text { ambos os grupos. } \\
\text { - A dispareunia diminuiu com o tempo e foi } \\
\text { baixa aos } 2 \text { grupos após } 6 \text { meses PP. }\end{array}$ & $\begin{array}{l}\text { Os escores de dor, } \\
\text { qualidade de vida e } \\
\text { ausência de dispareunia } \\
\text { foram melhores nas } \\
\text { primeiras } 6 \text { semanas } \\
\text { PP em mulheres que } \\
\text { fizeram extração a } \\
\text { vácuo, em comparação } \\
\text { com a cesárea. }\end{array}$ \\
\hline $\begin{array}{l}\text { Demaria et } \\
\text { al. } .^{13}\end{array}$ & $\begin{array}{l}\text { n: } 70 \text { puérperas; } \\
\text { Idade: > } 18 \text { anos; } \\
\text { PV: } 40 ; \\
\text { PC: } 10 ; \\
\text { PC e PV: } 3 \text {; } \\
\text { Não descrito: } 17 .\end{array}$ & Auto-relato & $\begin{array}{l}\text { - Ocorreu satisfação sexual no PP. } \\
\text { - Algumas dificuldades em RRS (dor ou } \\
\text { desconforto). } \\
\text { - A cesariana ou laceração dificultaram a RRS. }\end{array}$ & $\begin{array}{l}\text { Embora algumas } \\
\text { mulheres tenham } \\
\text { declarado que sua } \\
\text { libido diminuiu, a } \\
\text { satisfação sexual no } \\
\text { PP foi bem relatada. } \\
\text { Independentemente } \\
\text { do MP, as mulheres } \\
\text { tiveram experiências } \\
\text { diferentes na RRS. }\end{array}$ \\
\hline
\end{tabular}

Nota: FSFI: Índice de função sexual feminina; Questionário SF-12: Short-Form Health (questionário resumido de qualidade de vida); Escala de dor de McGill: Avaliação do padrão da dor; DF: Disfunção Sexual; PP: pós-parto; MP: Modo de Parto; PV: Parto Vaginal; PVE: Parto Vaginal Espontâneo; PC: Parto Cesáreo; PI: Parto Instrumental; FS: Função Sexual; RRS: Retomada da Relação Sexual. ENV: Escala Numérica Verbal; ECV: Escala de Classificação Verbal; EVA: Escala Visual Analógica.

Fonte: Elaborado pelos autores

\section{Discussão}

O objetivo deste estudo foi discutir a incidência e fatores de risco associados à dor sexual em puérperas, de um ponto de vista cinesiológico-funcional. Observou-se que a dor sexual é comum em mulheres que tiveram parto vaginal com episiotomia.

A dor sexual pode acometer, em média, de 30 a $50 \%$ das puérperas. ${ }^{12}$ Além disso, essa dor impacta negativamente na vida das puérperas, sendo que essa dor tende a perdurar para a maioria das mulheres por entre 6 a 12 meses. ${ }^{13,14}$

O puerpério é o período de muitas incertezas para a mulher. A retomada à prática sexual é uma delas. Nesse sentido, o fator psicológico está correlacionado também com a ausência ou presença de dor ou desconforto na relação sexual. ${ }^{14}$ Sendo assim, o puerpério não causa dispareunia. $\mathrm{O}$ que pode alavancar esse quadro é a utilização, 
por exemplo, de instrumentos no momento do parto como fórceps, os modos de episiotomia, lacerações perineais, extração à vácuo, dificultando a recuperação adequada da musculatura pélvica após o parto, afirmado nos artigos realizados por Demaria $e t$ al. ${ }^{13}$, com 70 mulheres; Lagaert et al. ${ }^{12}$, com 78 primíparas.

Outrossim, os principais fatores de risco para o aparecimento da dispareunia no puerpério são: problemas de cunho psicológico como depressão pós-parto e ansiedade, constipação e incontinência urinária. Além disso, segundo estudos realizados por Banaei e Dashti ${ }^{16}$ com 420 puérperas e Alligood-Percoco ${ }^{17}$, outros fatores como a amamentação, a intimidade entre o casal, casamento, número de filhos, primiparidade e multiparidade também podem ser considerados como fatores que influenciam na melhora da função sexual pós-parto, diminuindo a sua incidência dessa condição.

Os artigos entraram em contrassenso sobre a relação do tipo de parto com a dispareunia. Os estudos feitos por Kahramanoglu et al. ${ }^{18}$, realizado com 403 nulíparas apontam que muitas parturientes escolhiam a cesariana justamente para não apresentar problemas futuros durante a relação sexual. No entanto, o estudo de Kabakian-Khasholian et al. ${ }^{19}$, com 238 mulheres, afirma que houve uma incidência maior de disfunções sexuais em mulheres que tiveram a cesariana. Portanto, o que deve ser levado em consideração não é a via de parto, e sim a preparação da força dos músculos do assoalho pélvico na gravidez, a experiência da mulher com a retomada da relação sexual, os instrumentos utilizados no parto (fórceps, auxílio de vácuo) e o esclarecimento sobre a função sexual pós-parto entre a puérpera com a equipe multiprofissional.

Dentre os fatores etiológicos possivelmente relacionados com a dor sexual no pós-parto, o aleitamento materno foi descrito em cinco estudos. ${ }^{19}$ Ademais, a dor na relação sexual observada em seis semanas pós-parto está significativamente associada ao aleitamento materno. ${ }^{20}$ Esses dados corroboram com o estudo de Alligood-Perco$\mathrm{Co}^{17} \mathrm{em}$ que a prevalência de dispareunia em lactantes aos seis meses de pós-parto foi de $31,5 \%$, contra $12,7 \%$ de dispareunia entre as mulheres que não estavam amamentando. Em contrapartida, Fodstad, Staff e Laine ${ }^{22}$, não observaram associação entre dispareunia e amamentação, mas encontraram como causa da dor outros fatores como a laceração, episiotomia e uso de instrumento no parto.

Há divergências entre os estudos encontrados quanto à disfunção sexual e à via de parto. Há estudos que mostraram que as mulheres com parto cesáreo estiveram mais propensas a ter dor na relação sexual do que as de parto vaginal. ${ }^{22}$ Em contrapartida, há estudos que confirmam que a dor na relação sexual, a pior função sexual geral e a maior degeneração sobre a função orgástica prevalecem no parto vaginal. ${ }^{23}$ Nota-se, porém, que tanto o parto vaginal espontâneo com períneo íntegro como o parto cesáreo sempre se apresentam melhores que os partos com instrumentos, laceração ou com episiotomia. Todavia, um parto que necessita de instrumentalização, por si só, já é um parto que complicou e, desse modo, a quantidade de sequelas ou problemas associados é maior. Dentro de 6 e 12 semanas pós-parto a maioria das mulheres retornam à atividade sexual $^{24}$, mas uma grande parte das mesmas 
relatam sentir dor em sua primeira relação sexual vaginal após o parto. ${ }^{25,26}$

Pode- se perceber evidência e consonância entre todos os estudos de que a função sexual feminina degenera após o parto, sendo gradualmente recuperada, na maior parte dos casos, em até um ano após o parto. Os estudos encontrados que usaram o FSFI (Dabiri et al. ${ }^{14}$; Chayachinda et al. ${ }^{25}$; Ahmed et al. ${ }^{20}$; Necesalova et al..$^{33}$; Lagaert et al. ${ }^{12}$ ) que mediu a função sexual utilizou o questionário com escores de corte para disfunção sexual feminina chamado FSFI (Índice de Função Sexual Feminina) $)^{27}$, traduzido internacionalmente para quase todos os idiomas, o que facilitou a compilação desses dados. Mais especificamente podemos observar que após o parto ocorre uma piora significativa dos escores gerais que denotam a função sexual como um todo ${ }^{28}$, contudo essa disfunção tende a melhorar até os seis meses em comparação ao pré-natal, em especial as mulheres que tiveram parto cesáreo principalmente no escore de desejo sexual. ${ }^{29}$

De fato, há um aumento da probabilidade de três vezes mais chance de dor na relação sexual nas mulheres que passaram por parto vaginal instrumentalizado em relação ao parto vaginal não instrumentalizado ou parto cesáreo. ${ }^{30}$ Todavia, não houve diferença significativa na dor sexual posterior ao parto instrumentalizado e o cesáreo, mulheres com parto vaginal com instrumentos voltaram antes para as atividades sexuais, contudo o estudo não ressaltou nenhuma justificativa para tais achados como, por exemplo, o medo de sentir a dor que as mulheres das quais passaram pela cesárea possam vir a sentir. ${ }^{31}$ Outrossim, não houve associação entre as diferentes técnicas de episiotomia e a retomada do intercurso sexual, nem entre as episiotomias de curta ou longa duração com a retomada do coito. ${ }^{32} \mathrm{Da}$ mesma forma, não obteve diferença entre os grupos de incisão medial, lateral e não classificável com a retomada do ato sexual, no entanto este estudo usou como instrumento a escala de dor analógica (EVA) e questionamentos sobre a dispareunia. ${ }^{33}$

Há evidências de que a paridade está associada à dor sexual no pós-parto. ${ }^{34,35}$ Todos os demais demonstraram que as primíparas apresentaram pior função sexual no pós-parto, especialmente a dor, quando comparadas a multíparas. ${ }^{36}$

A depressão ou sintomas depressivos também foram correlacionados à dor sexual ${ }^{36}$; além disso, a dor na relação sexual e laceração grave do períneo são fatores de risco para a depressão. ${ }^{37}$

Quanto aos traumas perineais como consequência do parto vaginal, 13 estudos versaram sobre a laceração e 16 sobre episiotomia, sendo que ambos os traumas estiveram diretamente associados à dispareunia no pós-parto, apresentando escores que indicaram pior função sexual ${ }^{37,38}$, além de degeneração nos domínios desejo, orgasmo e satisfação sexual pelo FSFI. A soma desses fatores interfere no retorno da atividade sexual dessas mulheres. ${ }^{38}$ Por fim, os partos com episiotomia obtiveram maior prevalência de dispareunia com $40,4 \%$ em sete semanas e $27 \%$ em seis meses contra $32,9 \%$ em sete semanas e $17,8 \%$ em seis meses no grupo com lacerações. ${ }^{39,40}$

\section{Conclusão}

Partos vaginais estiveram mais relacionados à presença de dor sexual, es- 
pecialmente aqueles nos quais houveram grandes lacerações, com episiotomia ou instrumentalizado. Todavia, é importante ressaltar que um parto que evolui com instrumentos ou laceração já é um parto que pode causar graves consequências à mulher. Por outro lado, mesmo partos vaginais com períneo íntegro ou parto cesáreo também podem desencadear sintomas de dor sexual. Por fim, mulheres com dor sexual no pré-natal apresentam duas vezes mais chances de desenvolver dor sexual no pós-parto, apontando que a gestação em si é fator de risco para a dor sexual. Esse, portanto, é um tema que merece atenção científica urgente.

\section{REFERÊNCIAS}

1. Vettorazzi J, Marques F, Hentschel H, Ramos JGL, Costa SHM, Badalotti M. Sexualidade e puerpério: uma revisão da literatura. Rev. Hcpa. 2012; 32(4): 473-9

2. Holanda JBL, Abuchaim ESV, Coca KP, Abrao ACFV. Disfunção sexual e fatores associados relatados no período pós-parto. Acta paul. enferm. 2014; 27(6): 573-8

3. Moura TR, Nunes EFC, Latorre GFS, Vargas MM. Dispareunia relacionada à via de parto: uma revisão integrativa. Rev. ciênc. méd. 2019; 27(3): 157-65

4. Latorre GFS, Bilck PA, Cardoso FL, Sperandio FF. Validade e confiabilidade de uma versão on-line do Female Sexual Function Index por teste e reteste. Rev. Bras. Ginecol. Obstet. [online]. 2013; 35(10): 469-74

5. Benute GRG, Nomura RY, Santos AM, Zarvos MA, Lucia MCS, Francisco RPV. Preferência pela via de parto: uma comparação entre gestantes nulíparas e primíparas. Rev. Bras. Ginecol. Obstet. 2013; 35(6): 281-5

6. Mcdonald EA, Gartland D, Small R, Brown SJ. Dyspareunia and childbirth: a prospective cohort study. BJOG. 2015; 122(5): 672-9

7. Silva NLS, Oliveira SMJV, Silva FMB, Santos JO. Dispareunia, dor perineal e cicatrização após episiotomia. Rev. enferm. UERJ. 2013; 21(2): 216-20

8. Silva AP, Montenegro ML, Gurian MB, Mitidieri AM, Lara LA, Poli-Neto OB, et al. Perineal Massage Improves the Dyspareunia Caused by Tenderness of the Pelvic Floor Muscles. Rev Bras Ginecol Obstet. 2016; 39(1): 26-30

9. Eid MA, Sayed A, Abdel-Rehim R, Mostafa T. Impact of the mode of delivery on female sexual function after childbirth. Int J Impot Res. 2015; 27(3): 118-20

10. Latorre GFS, Manfrefini CCM, Demterco PS, Barros VMN, Nunes EFC. A .fisioterapia pélvica no tratamento da vulvodínia: revisão sistemática. Femina. 2015; 43(6): 257-64

11. Tomen A, Fracaro G, Nunes ECF, Latorre GFS. A fisioterapia pélvica no tratamento de mulheres portadoras de vaginismo. Rev. Ciênc. Méd. 2015; 24(3):121-30

12. Lagaert L, Weyers S, Van Kerrebroeck H, Elaut E. Postpartum dyspareunia and sexual functioning: a prospective cohort study. Eur J Contracept Reprod Health Care. 2017; 22(3): 200-6

13. DeMaria AL, Delay C, Sundstrom B, Wakefield AL, Avina A, Meier S. Understanding women's postpartum sexual experiences. Cult Health Sex. 2019; 21(10): 1162-76

14. Dabiri F, Yabandeh AP, Shahi A, Kamjoo A, Teshnizi SH. O Efeito do modo de entrega no pós-parto em primíparas. Funcionamento Sexual Feminino. OmanMed J. 2014; 29(4):276-9

15. Mcdonald E, Woolhouse H, Brown SJ. Consultation about Sexual Health Issues in the Year after Childbirth: A Cohort Study. Birth. 2015; 42(4): 354-61 
16. Banaei M, Dashti A. Sexual Dysfunction and its Associated Factors After Delivery: Longitudinal Study in Iranian Women. Mater Sociomed. 2018; 30(2): 198-203

17. Alligood-percoco NR, Kjerulff KH, Repke JT. Risk Factors for Dyspareunia After First Childbirth. Obstet Gynecol. 2016; 128(3): 512-8

18. Kahramanoglu I, Baktiroglu M, Hamzaoglu K, Kahramanoglu O, Verit FF, Yucel O. The impact of mode of delivery on the sexual function of primiparous women: a prospective study. Arch Gynecol Obstet. 2017; 295(4): 907-16

19. Kabakian-Khasholian T, Ataya A, Shayboub R, El-Kak F. Mode of delivery and pain during intercourse in the postpartum period: Findings from a developing country. Sex Reprod Healthc. 2015; 6(1): 44-7

20. Sayed Ahmed WA, Kishk EA, Farhan RI, Khamees RE. Female sexual function following different degrees of perineal tears. Int Urogynecol J 2016; 28(6): 917-921.

21. Amiri FN, Omidvar S, Bakhtiari A, Hajiahmadi M. Female sexual outcomes in primiparous women after vaginal delivery and cesarean section. Afr Health Sci. 2017; 17(3): 623-31

22. Fodstad K, Staff AC, Laine K. Effect of different episiotomy techniques on perineal pain and sexual activity 3 months after delivery. Int Urogynecol J. 2014; 25(12): 1629-37

23. McDonald EA, Gartland D, Small R, Brown SJ. Frequency, severity and persistence of postnatal dyspareunia to 18 months post partum: A cohort study. Midwifery, 2016; 34: 15-20.

24. De Souza A, Dwyer PL, Charity M, Thomas E, Ferreira CH, Schierlitz L. The effects of mode delivery on postpartum sexual function: a prospective study. BJOG. 2015; 122(10): 1410-8

25. Chayachinda C, Titapant V, Ungkanungdecha A. Dyspareunia and Sexual Dysfunction after Vaginal Delivery in Thai Primiparous Women with Episiotomy. The J Sex Med. 2015; 12(5): 1275-82

26. Aratani N, Toledo JL Neto, Silva NMM, Tashima CM, Melo SCC, Marzola C. Preferência do tipo de parto entre gestantes primíparas.Rev. Odontologia (ATO). 2014; 14(3): 209-24

27. Barbara G, Pivarotti P, Facchin F, Cortinovis I Dridi D, Ronchetti C, et al. Impact of Mode of Delivery on Female Postpartum Sexual Functioning: Spontaneous Vaginal Delivery and Operative Vaginal Delivery VS Cesarean Section. J Sex Med. 2016; 13(3): 393-401

28. Puppo V. Anatomy and Physiology of the Clitoris, Vestibular Bulbs, and Labia Minora With a Review of the Female Orgasm and the Prevention of Female Sexual Dysfunction. Clin Anat. 2013; 26(1):134-52

29. Chang SR, Chen KH, Lin HH, Chao YM, Lai YH. Comparison of the effects of episiotomy and no episiotomy on pain, urinary incontinence, and sexual function 3 months postpartum: A prospective follow-up study. Int J Nurs Stud. 2011; 48(4): 409-18

30. Persico G, Vergani P, Cestaro C, Grandolfo M, Nespoli A. Assessment of postpartum perineal pain after vaginal delivery: prevalence, severity and determinants. A prospective observational study. Minerva Ginecol. 2013; 65(6): 669-78

31. Norhayati MN, Yacob MA. Long-term postpartum effect of severe maternal morbidity on sexual function. Int J Psychiatry Med. 2017; 52(4): 328-44

32. Nolens B, Akker TV, Lule J, Twinomunhangi S, Roosmalen JV, Byamugisha J. Birthing experience and quality of life after vacuum delivery and second-stage caesarean section: a prospective cohort study in Uganda. Trop Med Int Health. 2018; 23(8): 914-22

33. Necesalova P, Karbanova J, Rusavy Z, Pastor Z, Jansova M, Kalis V. Mediolateral versus lateral episiotomy and their effect on postpartum coital activity and dyspareunia rate 3 and 6 months postpartum. Sex Reprod Healthc. 2016; 8: 25-30.

34. Chang SR, Chen KH, Ho HN, Lai YH, Lin M, Lee CN, et al. Depressive symptoms, pain, and sexual dysfunction over the first year following vaginal or cesarean delivery: A prospective longitudinal study. Int J Nurs Stud.Nursing. 2015; 52(9):1433-44 
35. Chang S, Lin W, Lin H, Shyu M, Lin M. Sexual dysfunction predicts depressive symptoms during the first 2 years postpartum. Women Birth. 2018; 31(6): 403-11

36. Chivers ML, Pittini R, Grigoriadis S, Villegas L, Ross LE. The Relationship between Sexual Functioning and Depressive Symptomatology in Postpartum Women: A Pilot. Study. J Sex Med. 2011; 8(3): 792-9

37. Huang LH, Lai YF, Chen GD, Lee MS, Ng SC. Effect of far-infrared radiation on perineal wound pain and sexual function in primiparous women undergoing an episiotomy. Taiwan J Obstet Gynecol. 2019; 58(1): 68-71.

38. Latorre GFS, Manfredini CCM, Demterco PS, Barreto VMNF, Nunes EFC. A fisioterapia pélvica no tratamento da vulvodínia: revisão sistemática. Femina. 2015; 43(6): 257-64

39. Leeman L, Rogers R, Borders N, Teaf D, Qualls C. The Effect of Perineal Lacerations on Pelvic Floor Function and Anatomy at 6 Months Postpartum in a Prospective Cohort of Nulliparous Women. Birth. 2016; 43(4): 293-302

40. Sargin MA, Yassa M, Taymur BD, Taymur B, Akca G, Tug N. Female Sexual Dysfunction in the Late Postpartum Period Among Women with Previous Gestational Diabetes Mellitus. J Coll Physicians Surg Pak. 2017; 27(4): 203-8

\section{DADOS DOS AUTORES}

Ariana F. dos S. Bartolomey Mendes

Fisioterapeuta auntonoma. arianabartolomeyfisio@gmail.com

\section{Paula Ferrarezi Brunazzo}

Fisioterapeuta autonoma. paulafbrunazo@gmail.com

\section{Fernanda Morais Silva}

Fisioterapeuta autonoma. fernandamorais.fisio@gmail.com

\section{Brenda Beatriz Silva Monteiro}

Estudante na Universidade do Estado do Pará, Belém/PA - Brasil.

\section{Erica feio Carneiro Nunes}

Fisioterapeuta. Docente na Universidade do Estado do Pará, Belém/PA - Brasil. ericacarneiro@uepa.br

\section{Gustavo Fernando Sutter LAtorre}

Fisioterapeuta. Professor da Gustavo Latorre Cursos. gustavo@perineo.net

Submetido em: 23-12-2020

Aceito em: 29-4-2021 